\title{
Computer Aided Detection of Obstructive SLEEP APNEA FROM EEG SIGNALS
}

\author{
Saheed Ademola Bello and Umar Alqasemi
}

King AbdulAziz University, Jeddah, Saudi Arabia

\begin{abstract}
Sleep Apnea is an anomaly in sleeping characterized by short pause in breathing. Failure to treat sleep apnea leads to fatal complications in both psychological and physiological being of human. Electroencephalogram (EEG) performs an important task in probing for sleep apnea through identifying and recording the brain's activities while sleeping. In this study, computer aided detection of sleep apnea from EEG signals is developed to optimize and increase the prompt recognition and diagnosis of sleep apnea in patients. The time domain, wavelets, and frequency domain of the EEG signals were computed, and features were extracted from these domains. These features are inputted into two machine learning algorithms: Support Vector Machine and K-Nearest Neighbors of different kernel functions and orders. Evaluation metrics such as specificity, accuracy, and sensitivity are computed and analyzed for the classifiers. The KNN classifier outperforms the SVM in classifying apnea from non-apnea events in patients. The KNN order 3 shows the highest performance sensitivity of $85.92 \%$, specificity of $80 \%$ and accuracy of $82.69 \%$.
\end{abstract}

\section{KEYWORDS}

Signal Processing, Computer Aided Detection, Obstructive Sleep Apnea, EEG signal, Support Vector Machine.

\section{INTRODUCTION}

Sleep is a state of rest and part of a daily rhythm essential for human life which covers about thirty- three percent (33\%) of human existence[1].Sleep is an important part of human existence that helps in regulating mental and physical activities [2]. Sleeping leads to decrease in blood pressure, heart rate and body metabolic rate. It also helps in the relaxation of muscles, repair and replacement of dead cells and tissues. Also, sleep gives the brain a chance to organize and fix memories. The significance of a good sleep cannot be overemphasized, yet a lot of people experiences sleep disorders. National Sleep Foundation reveals that two-third of adults reported experiencing sleep disorders at night, a couple of times in a week or more. Furthermore, more than one-third of adults also experiences severe daytime drowsiness at least a few days each month[3].

A sleep disorder is an inability to sleep, thereby, leading to deficiency in body function both physically and mentally. Currently, there are about eighty-four different sleep disorders affecting human like sleep apnea, Parasomnias, Periodic limb movement disorder, insomnia, and narcolepsy [4].

Sleep Apnea (SA) is the disease characterized with pauses or shortness in breathing while asleep[5]. Sleep Apnea is of three types; Obstructive sleep apnea (OSA) is produced by total closure or fall of the human throat while sleeping. It occurs due to the relaxation of the sensitive tissues at the end of the throat. It is the most common kind of sleep apnea, and it also affects all 
Signal \& Image Processing: An International Journal (SIPIJ) Vol.12, No.3, June 2021

ages of mankind[1]. Central Sleep Apnea (CSA) occurs due to breakdown in the nervous system. The organ responsible for the control of breathing could not maintain the breathing process. As a result, activation signals from the brain could not reach the necessary muscles. It seldomly occurs and it often has a neural cause[1]. Mixed Sleep Apnea (MSA) consists of mixture of obstructive apnea and central apnea.

According to [6] on cases of OSA in America, 22 million people experience sleep apnea with $80 \%$ of incidents going undetected on time. It was studied that that $25 \%$ of middle-aged men has OSA, about $3 \%$ of children suffer from OSA and apnea patients are highly prone to having Stroke. Sleep apnea leading to cardiovascular failure leads to over 38,000 deaths yearly. The total cost of diagnosing and treating sleep apnea is over $\$ 65$ billion. Polysomnography (PSG) is a sleep disorder diagnostic test, performed in a sleep center where different physiological quantities are measured and documented during sleep. The signals were obtained directly by electrodes and then amplified. The PSG signalswere scored offline by sleep experts [7]. These signals contain of: Electromyogram (EMG); Electrooculogram (EOG), Electrocardiogram (ECG) and Electroencephalogram (EEG) [7].

Polysomnography (PSG) is usually studied and scored manually to detect, but this approach is challenging because of two reasons. Firstly, the high cost of PSG and having patients to sleep in an unaccustomed environment of sleep centers degrades the quality of sleep. Secondly, the physical scoring of OSA consumes time, and its' efficiency is highly dependent on human expertise and experience [8]. Consequently, large datasets can't be handled effectively using the manual detection of OSA. Hence, a computer aided diagnosis of OSA is imperative.

Electroencephalogram (EEG) are recordings of the electrical activities of the brain. It has been studied that sleep apnea can be detected by monitoring brain activities [9]. Many sleep studies show that sleep disorders can be identified and predicted through channels (C3-A2 channel or C4-A1 channel) of the EEG signal [9].In this research, we are going to develop a system that automatically recognizes apnea events in patients using the time domain, wavelets, and frequency domains of EEG signals. Features were extracted from these domains and fed into classifiers.

\section{LiterATURE REVIEW}

Different works has been done in the automatic or computer aided detection of obstructive sleep apneas. Features from different signals such as acoustic speech signal, abdomen effort and thorax signals, nasal air flow, oxygen saturation (Sp02), EEG, and ECG are commonly used as yardsticks for detecting sleep apnea.

The acoustic properties in human are affected by the vocal tract structure and soft tissue found in the throat. It was proposed by [10] that speech signal characteristics of OSA patients were different from the Non-OSA patients. A Gaussian Mixture Model (GMM) system was developed, relying on the vocal tract length and the linear prediction coefficients as features. An $83 \%$ Specificity, $79 \%$ sensitivity for male patients and $86 \%$ specificity, $84 \%$ sensitivity for female patients were achieved. In [11], $80.65 \%$ sensitivity and $80 \%$ specificity were achieved using the GMM-based system classifier corroborated with a Mel Frequency Cepstral Coefficients (MFCC) feature space.

[12] used the signals of abdominal and thoracic efforts, oral and nasal airflow, and pulse oximetry level to indicate the presence of apnea. Using mean absolute amplitude analysis, the results showed that combining the abdominal and thoracic signals achieved $80 \%$ sensitivity and $90 \%$ specificity. 
Signal \& Image Processing: An International Journal (SIPIJ) Vol.12, No.3, June 2021

Features extracted ECG provide an efficient means of detecting sleep disorders. [7] used the RRInterval of the ECG signals as features inputted. The results showed that system detected epochs of apnea with an accuracy of 96.5\%. A single-lead ECG signal of 1-min segment is disintegratedinto frequency bands using Fast Fourier Transform (FFT) in [13]. The computed mean absolute deviation and entropy were used to classify ECG segments. 92.59\% accuracy was gotten when it was implemented on a Gaussian kernel SVM classifier.

Relying on the statistical and spectral features, [8] employed the use of bootstrap aggregating for the classification of a single -lead ECG, the method yielded $84.14 \%$ sensitivity, $86.83 \%$ specificity, and $85.97 \%$ accuracy. In [14], the extracted features of arterial oxygen saturation signal (Sp02) measured in PSG and the ECG are combined to identify apnea episodes. These features are used to train, test, and validate a neural network, a high accuracy of $93.3 \%$ was gotten.

The discrete wavelet transform of ECG, EMG, and EEG signals were computed in [15] to achieve a time -frequency bands and the transform was fed into a Multilayer Perceptron (MLP). 96.87\% Specificity, $97.41 \%$ sensitivity, and $98.9 \%$ accuracy were obtained through combining EMG, ECG and EEG signals. The use of fuzzy set turning algorithm was developed by [16] to identify apnea conditions. Though the method performed below MLP in the wekalibrary Weka is an open source, public machine learning workbench, it exceeded other methods in the library.

In the study of [9], the EEG signals were filtered and disintegrated using the Infinite Impulse Response (IIR) Butterworth band-pass filters. The extracted features like the energy and variance were used as the bases for classification on the SVM, artificial neural networks, linear discriminant analysis and naïve bayes. The system developed had the highest accuracy of $97.14 \%$ on the SVM. In [17], the EEG signals was filtered by Infinite Impulse Butterworth Band Pass filter and Hilbert Huang Transform. Extracted features like energy, variance and entropy were fed into classifiers like SVM, Artificial Neural Network, and K-Nearest Neighbors.

Entropy of the frequency band- limited EEG signals was the only feature of classifying apnea in [18]. The frequency band- limited signals was gotten by fast Fourier transform (FFT), then entropy of each frequency band limited EEG signals were then extracted, cascaded, and classified using KNN classifier. The result showed a mean accuracy of $87.64 \%$ over a database. While improving on this method, [19] proposed the use of subframes from the EEG signals in place of the earlier full frame and the use of temporal variation in the beta band energy is used for classifying the apnea events in the KNN classifier. The performance increased significantly.

Studies has also been done in the area of applying deep learning to EEG signals to automatically detect apnea events, [20] used the concept of dividing frames of EEG signals into subframes. Each subframeis fed into a fully convolutional neural network (FCNN). The feature vectors of each subframeare concatenated together and fed to a dense classifier (deep neural network) to generate the final features for detecting apnea and non-apnea events. A mean accuracy of $77.14 \%$ was achieved. 
Signal \& Image Processing: An International Journal (SIPIJ) Vol.12, No.3, June 2021

Table 1 shows a summary of past research results

\begin{tabular}{|c|c|c|c|c|c|c|c|}
\hline \multirow[t]{2}{*}{$\mathrm{S} / \mathrm{N}$} & \multirow{2}{*}{$\begin{array}{l}\text { Author's } \\
\text { Name }\end{array}$} & \multirow{2}{*}{$\begin{array}{l}\text { Features } \\
\text { used }\end{array}$} & \multirow[t]{2}{*}{ Classifiers } & \multirow[t]{2}{*}{ Signal \& Dataset } & \multicolumn{3}{|c|}{ Performance } \\
\hline & & & & & $\begin{array}{l}\mathrm{Se} \\
(\%)\end{array}$ & $\begin{array}{l}\mathrm{Sp} \\
(\%)\end{array}$ & $\begin{array}{l}\text { Acc } \\
(\%)\end{array}$ \\
\hline 1 & [9] & $\begin{array}{l}\text { Energy, } \\
\text { Variance }\end{array}$ & $\begin{array}{l}\text { SVM, KNN, } \\
\text { ANN, LDA, } \\
\text { Naïve Bayes }\end{array}$ & $\begin{array}{c}\text { EEG/ MIT-BIH } \\
\text { Polysomnography }\end{array}$ & 97 & 97 & 97 \\
\hline 2 & [17] & $\begin{array}{l}\text { Energy, } \\
\text { Entropy, } \\
\text { Variance }\end{array}$ & SVM, KNN & $\begin{array}{c}\text { EEG/ MIT-BIH } \\
\text { Database }\end{array}$ & 100 & 98 & 99 \\
\hline 3 & {$[18]$} & $\begin{array}{l}\text { Multiband } \\
\text { Entropy }\end{array}$ & KNN & $\begin{array}{l}\text { EEG/ St. Vincent } \\
\text { University Database }\end{array}$ & 89 & 86 & 88 \\
\hline 4 & [19] & $\begin{array}{l}\text { Inter-band } \\
\text { Energy }\end{array}$ & KNN & $\begin{array}{l}\text { EEG / St. Vincent } \\
\text { University Database }\end{array}$ & 90 & 94 & 92 \\
\hline 5 & [20] & Subframes & $\begin{array}{l}\text { Fully Connected } \\
\text { Neural Network }\end{array}$ & $\begin{array}{c}\text { EEG signal/ St. } \\
\text { Vincent University } \\
\text { Database }\end{array}$ & 77 & 78 & 76 \\
\hline
\end{tabular}

\section{Materials ANd Methodology}

\subsection{Database}

The EEG dataset was gotten from St. Vincent's University Hospital/ University College Dublin Sleep Apnea Database that is publicly accessible through Physionet [21]. This dataset contains the overnight polysomnography collected from 25 adult subjects who have been previously diagnosed with sleep disordered breathing. The PSG has been scored by sleep expert.

In this research, 5 patients of different apnea hypopnea index are considered. The information of these patients is provided in the table below. From the two EEG channels, C3-A2 channel is considered. The EEG signals are converted into 10-second frames, with a frame either containing apnea event or non-apnea event. For testing, equal number of apnea frames and non-apnea frames are used. The patient's information is provided in table 2.

Table 2: Patients Information

\begin{tabular}{|c|c|c|c|c|c|}
\hline $\begin{array}{c}\text { Subject } \\
\text { Number }\end{array}$ & AHI & Height $(\mathrm{cm})$ & Weight(kg) & Gender & $\begin{array}{c}\text { Total number of } \\
\text { frames }\end{array}$ \\
\hline UCDDB007 & 12 & 183 & 84 & $\mathrm{M}$ & 260 \\
\hline UCDDB011 & 8 & 188 & 101 & $\mathrm{M}$ & 126 \\
\hline UCDDB012 & 25 & 179 & 97.5 & $\mathrm{M}$ & 500 \\
\hline UCDDB013 & 16 & 153 & 80 & $\mathrm{~F}$ & 270 \\
\hline UCDDB024 & 24 & 172 & 99.9 & $\mathrm{M}$ & 400 \\
\hline
\end{tabular}

\subsection{Preprocessing}

The mean value of an EEG test frame is subtracted to remove the dc offset as components of other frequencies are of major interest. These EEG signals are in the time domain. The wavelet decomposition of these frames was done by applying a 1D-wavelet transform. The approximation coefficients and the detail coefficients are used for feature extraction. The Fast Fourier Transform of the EEG frames are also computed which also serve as input for the feature extraction. 


\subsection{Feature Extraction}

To enable accurate classification of sleep apnea, features are computed from the time domain, wavelet domain and frequency domain. Features used are Energy, Variance, Skewness, Mode, Standard Deviation, Median, geometric mean, Minimum, Kurtosis, Mean, Entropy, and Maximum. Table 3 below shows the formulae of these features.

Table 3: Formulae of some Features

\begin{tabular}{|c|c|c|c|}
\hline Features & Formulae & Features & Formulae \\
\hline Energy & $E=\sum_{i}^{N}\left|X_{i}^{2}\right|$ & Mean & $\bar{X}=\frac{1}{N} \sum X$ \\
\hline Entropy & $E N=\sum_{i=1}^{N} X_{i}^{2} \log X_{i}^{2}$ & Skewness & $s=\frac{\sum(X-\bar{X})^{3}}{\sigma^{3}}$ \\
\hline $\begin{array}{c}\text { Standard } \\
\text { deviation }\end{array}$ & $s t d=\sigma$ & Kurtosis & $k=\frac{\sum(X-\bar{X})^{4}}{\sigma^{4}}$ \\
\hline Variance & $=\sqrt{\frac{1}{N} \sum_{i=1}^{N}\left|X_{i}-\mu\right|}$ & & $g=\left[\prod_{i=1}^{n} x_{i}\right]^{\frac{1}{n}}$ \\
\hline
\end{tabular}

\subsection{CLASSIFICATION}

The features generated are used to classify between apnea events and non-apnea events. Two classifiers used are Support vector machine (SVM) and K-Nearest Neighbor (KNN) classifiers.

\section{A. Support Vector Machines (SVM)}

It is a type of supervised learning algorithms that can be used for classifications of data samples. the objective is to find a hyperplane in a $\mathrm{N}$-dimensional space ( $\mathrm{N}$ - the number of features) that distinctly classifies the data points by finding a plane that has the maximum margin. This margin is maximized by using suitable techniques provided by the kernel function. In this research, various kernel functions were used ranging from Radial Basis Function (RBF) kernel, Polynomial kernel, Gaussian kernel, and Linear kernel.

\section{B. K-Nearest Neighbor (KNN)}

It is another machine learning classifier algorithm that is used for classification and regression predictive problems. the major parameters used for the classifying include number of nearest neighbors and the distance metric. To achieve high performance, Euclidean distance and MahalaNobis distance are usually adopted. The distance function is computed using the KNN patterns developed from the training set and features extracted from the test set of the EEG signals. Based on the $\mathrm{K}$ closer patterns' class labels in the train set, the test set is classified. $K$ values from 1 to 5 are used in this research. 


\subsection{Performance Assessment Metrics}

The features extracted from the processed EEG signals are imported into the SVM and KNN classifiers. The performance metrics of these classifiers are evaluated using accuracy, sensitivity, and specificity.

The accuracy is defined by the classifier's ability to distinguish the apnea events from non-apnea events correctly. Accuracy is the number of correct predictions over the total number of predictions.

$$
\text { Accuracy }=\frac{T P+T N}{T P+T N+F P+F N}
$$

The sensitivity of this system is its ability to determine the Sleep Apnea cases correctly.

$$
\text { Sensitivity }=\frac{T P}{T P+F N}
$$

The specificity of this system is its ability to determine the Normal cases correctly.

Where,

$$
\text { Specficity }=\frac{T N}{T N+F P}
$$

True Positive (TP) is the number of cases correctly identified as Sleep Apnea,

False Positive (FP) is the number of normal cases incorrectly identified as Sleep Apnea,

True Negative (TN) is the number of cases correctly identified as Normal,

False Negative (FN) is the number of Sleep Apnea cases incorrectly identified as Normal.

\section{ReSults AND Discussions}

A computer aided system that classifies apnea events and non-apnea events using 5 patients was developed. The inputted signals were sampled at $128 \mathrm{~Hz}$ and divided into 10 -second frame. Features were extracted from the time domain, wavelet and frequency domain of these frames as discussed earlier. The performance of these classifiers was assessed using the accuracy, specificity, and sensitivity. $90 \%$ of the dataset was used to train the classifiers and $10 \%$ is used for testing. The whole process is done on MATLAB2021a environment. Table below shows the performance analysis of the eight classifiers used in this research with training set and testing set.

Table 4: Performance Analyses

a. Training Set

\begin{tabular}{|l|l|l|l|}
\hline Classifiers & Sensitivity & Specificity & Accuracy \\
\hline SVM with RBF kernel & 100 & 100 & 100 \\
\hline SVM with Polynomial kernel & 50 & Nan & 50 \\
\hline SVM with Gaussian kernel & 100 & 100 & 100 \\
\hline SVM with linear kernel & 80.51 & 63.57 & 68.79 \\
\hline KNN of order 1 & 100 & 100 & 100 \\
\hline KNN of order 2 & 79.91 & 100 & 87.43 \\
\hline KNN of order 3 & 87.59 & 86.01 & 86.79 \\
\hline KNN of order 4 & 81.12 & 89.61 & 84.86 \\
\hline KNN of order 5 & 85.69 & 80.93 & 83.14 \\
\hline
\end{tabular}


Signal \& Image Processing: An International Journal (SIPIJ) Vol.12, No.3, June 2021

b. Testing Set

\begin{tabular}{|l|l|l|l|}
\hline Classifiers & Sensitivity & Specificity & Accuracy \\
\hline SVM with RBF kernel & 50.32 & 100 & 50.64 \\
\hline SVM with Polynomial kernel & 50 & NAN & 50 \\
\hline SVM with Gaussian kernel & 50.32 & 100 & 50.64 \\
\hline SVM with linear kernel & 83.67 & 65.42 & 71.15 \\
\hline KNN of order 1 & 81.08 & 78.05 & 79.49 \\
\hline KNN of order 2 & 74.19 & 85.71 & 78.84 \\
\hline KNN of order 3 & 85.92 & 80 & 82.69 \\
\hline KNN of order 4 & 75.90 & 79.45 & 77.56 \\
\hline KNN of order 5 & 80.28 & 75.29 & 77.56 \\
\hline
\end{tabular}

From the performance analysis, it is seen that the KNN classifier performed best in the accurately clssifying the apnea event and non apnea event. With KNN of order 3 providing the best accuracy of $82.69 \%$. The SVM classifiers performed fairly in the classification with SVM of linear kernel showing promising result of $71.15 \%$ accuracy.

\section{Conclusion}

This study is focused on developing an automatic detection of obstructibve sleep apnea using EEG. The EEG signals was gotten from an open source[21] and the time domain, wavelets and frequency domain of the EEG signals are computed. Statistical features are computed from these domains and these features are used as bases for the classification of apnea events and non-apnea events. The KNN based classifiers outperform the SVM based classifiers with various order of the KNN showing high accuracy as opposed the SVM classifiers. The SVM classifier of linear kernel shows the highest accuracy of $71.15 \%$ amongst the SVM based classifier. The KNN of order 3 shows the highest accuracy of $82.69 \%$ amongst the eight classifiers used.

\section{REFERENCE}

[1] E. F. Estrada, "Computer-Aided Detection of Sleep Apnea and Sleep Classification using HRV and EEG Signals," DigitalCommons@UTEP, University of Texas at El Paso, 2010-01-01.

[2] E. Estrada,HNazeran, J Barragan,J R Burk, E A Licas, K Behbehani "EOG and EMG: Two Important Switches in Automatic Sleep Stage Classification," Proceedings of the 28th IEEE EMBS Annual International Conference, p. 2458, 2006.

[3] Ganesh Balakrishnan,DivyaBurli, John R Burk, Edgar A Lucas, KhosrowBehbehani "Comparison of a Sleep Quality Index between Normal and Obstructive Sleep Apnea Patients," Proceedings of the 2005 IEEE Engineering in Medicine and Biology 27th Annual Conference, p. 1154, 2005.

[4] WIKIPEDIA, "International Classification of Sleep Disorders," [Online]. Available: https://en.wikipedia.org/wiki/International_Classification_of_Sleep_Disorders\#: :text=In\%202005,\% 20the $\% 20$ International $\% 20$ Classification $\% 20$ of $\% 20$ Sleep $\% 20$ Disorders, in $\% 20 \mathrm{a} \% 20 \mathrm{description} \% 20 \mathrm{t}$ hat\%20presents\%20the\%20diagnostic\%20criteria.. [Accessed 4 feb 2021].

[5] Sani M. Isa, Mohammad Ivan Fanny, WisnuJatmiko, AniatoMurniArymurthy "Sleep Apnea Detection from ECG Signal, Analysis on Optimal Features, Principal Components, and Nonlinearity," in Proceedings of the 5th IEEE International Conference on Bioinformatics and Biomedical Engineering (iCBBE, pp 1-4, May 2011.

[6] M. Rakicevic , "31 Important Sleep Apnea Statistics You Should Know in 2021," 08 December 2020. [Online]. Available: https://disturbmenot.co/sleep-apnea-statistics/. [Accessed 12 Febuary 2021].

[7] L. Almazaydeh, K. Elleithy and M. Faezipour, "Obstructive Sleep Apnea Detection Using SVMBased Classification of ECG Signal Features," in 34th Annual International Conference of the IEEE EMBS, San Diego, California USA, 1 Sept, 2012. 
Signal \& Image Processing: An International Journal (SIPIJ) Vol.12, No.3, June 2021

[8] A. R. Hasan and M. A. Haque, "Computer-aided obstructive sleep apnea screening from single-lead electrocardiogram using statistical and spectral features and bootstrap aggregating," ELSEVIER : BIOCYBERNETICS AND BIOMEDICAL ENGINEERING, vol. 36, pp. 256-266, 2016.

[9] W. S. Almuhammadi, M. Faezipour and k. A. Aboalayon, "Efficient Obstructive Sleep Apnea Classification Based on EEG signals," IEEE, Vols. 978-1-4577-1343-9, pp. 1-6, 2015.

[10] E. Goldshtein, A. Tarasiuk and Y. Zigel, "Automatic Detection of Obstructive Sleep Apnea using Sound Signals," IEEE TRANSACTIONS ON BIOIMEDICAL ENGINEERING, vol. 58, p. 1373, 2011.

[11] M. Kriboy, A. Tarasiuk and Y. Zigel, "OBSTRUCTIVE SLEEP APNEA DETECTION USING SPEECH SIGNALS," IEEE, vol. 65, p. 1874, 2013.

[12] A. S. Ng, J. W. Chung, M. D. Gohel, W. W. Yu, K. L. Fan and T. K. Wong, "Evaluation of the performance of using mean absolute amplitude analysis of thoracic and abdominal signals for immediate indication of sleep apnoea events," Journal of Clinical Nursing , Vols. doi: 10.1111/j.1365-2702.2008.02323.x, p. 2360, December, 2008.

[13] F. Binish, P. Singh, A. Singhal and R. B. Pachori, "Detection of apnea events from ECG segments using Fourier decomposition method," Biomedical Signal Processing and Control, vol. 61, pp. 1-6, 2020.

[14] L. Almazaydeh, F. Miad and E. khaled, "A Neural Network System for Detection of Obstructive Sleep Apnea Through SpO2 Signal Features," International Journal of Advanced Computer Science and Applications, vol. 3, no. No 5, pp. 18-23, 2012.

[15] M. K. Moridani, M. Heydar and S. S. J. Behnam, "A Reliable Algorithm Based on Combination of EMG, ECG and EEG Signals for Sleep Apnea Detection," in 5th Conference on Knowledge-Based Engineering and Innovation, Iran, 2019.

[16] D. E. Dow and I. Hayashi, "Fuzzy Set Training for Sleep Apnea Classification," in IFSA-SCIS, Otsu,Shiga,Japan, 2017.

[17] V. Vimala, K. Ramar and M. Ettapan, "An Intelligent Sleep Apnea Classification System Based on EEG Signals," Journal of Medical Systems, vol. 36, pp. 1-9, 2019.

[18] S. Saha, A. Bhattacharjee, M. A. A. Ansary and S. Fattah, "An Approach for Automatic Sleep Apnea Detection Based on Entropy of Multi-Band EEG Signal," IEEE, vol. 16, no. 978-1-5090-2597-8, p. 420, 2016.

[19] F. Ahmaed, P. Paromita, A. Bhattacharjee, S. Saha, S. Azad and S. Fattah, "Detection of Sleep Apnea Using Sub-frame Based Temporal Variation of Energy in Beta Band in EEG," in 2016 IEEE International WIE Conference on Electrical and Computer Engineering (WIECON-ECE), Pune,India, 2016.

[20] Tanvir Mahmud, I. A. Khan, Talha Ibn Mahmud, S. A. Fattah, Wei-Ping Zhu, and M. O. Ahmad, "Sleep Apnea Event Detection from Sub-frame Based Feature Variation in EEG Signal Using Deep Convolutional Neural Network," IEEE, vol. 20, no. 978-1-7281-1990-8, p. 5579, 2020.

[21] W. McNicholas, L. Doherty, S. Ryan, J. Garvey, P. Boyle and E. Chua, "St. Vincent University Hospital/ University College Dublin Sleep Apnea Database," Physionet.org, 2004. [Online]. Available: https://physionet.org/content/ucddb/.

\section{AUTHORS}

Engr. Saheed Ademola Bello is a graduate student of computer engineering at King AbdulAziz University, Jeddah, Saudi Arabia. He obtained his Bachelor's degree at Obafemi Awolowo University, Ile-Ife, Nigeria in the same field. His research interests are computer vision and machine learning.

Dr. Umar S. Alqasemi, Associate Professor of Biomedical Engineering at the Dept. of Electrical and Computer Engineering, King, Abdulaziz University, Jeddah 21589, Saudi Arabia. PhD and MSc degree in Biomedical Engineering from UConn, Storrs, USA. Research work in ultrasound, optical, and photoacoustic imaging, medical imaging recognition, bioelectronics, and digital and analog signal and image processing
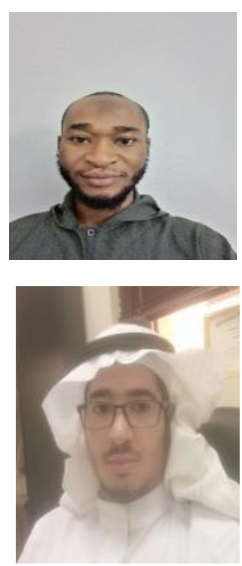